\title{
REFLEXÕES ACERCA DO DESEMPENHO E DAS DIFICULDADES DE ESTUDANTES DA EDUCAÇÃO BÁSICA E SUPERIOR NAS OPERAÇÕES COM FRAÇÕES
}

\author{
REFLECTIONS ON THE PERFORMANCE AND DIFFICULTIES OF \\ STUDENTS IN BASIC AND HIGHER EDUCATION WITH OPERATIONS WITH \\ FRACTIONS
}

\author{
Teresa Cristina Etcheverria \\ Universidade Federal de Sergipe - UFS \\ tetcheverria@gmail.com \\ Viviane de Jesus Lisboa Aquino \\ Universidade Federal de Sergipe - UFS \\ vjlisboa@yahoo.com.br \\ Jackeline dos Santos Oliveira \\ Universidade Federal de Sergipe - UFS \\ jacky_oliveira@yahoo.com.br \\ Caroline de Carvalho Lisboa \\ Escola Municipal Maria Eloiza Batista Santos \\ caroline.c.lisboa@hotmail.com
}

\section{Resumo}

Este texto tem como objetivo discutir o desempenho e as dificuldades apresentadas por estudantes da educação básica e por graduandos do curso de Licenciatura em Matemática na resolução das quatro operações fundamentais com frações. Para esta discussão nos apoiamos nas ideias de Llinares e Sánchez (1988), Monteiro e Groenwald (2014) e Silva e Almouloud (2008), dentre outros. Os dados foram coletados por meio da aplicação de um questionário a 358 estudantes de turmas de $7^{\circ}$ e $9^{\circ}$ ano do ensino fundamental, $1^{\circ}$ e $3^{\circ}$ ano do ensino médio e graduandos de Cálculo I e II do Curso de Licenciatura em Matemática. Os resultados mostram que o índice de desempenho dos estudantes em cada uma das operações foi insatisfatório, apesar de evidenciar crescimento conforme o nível de escolaridade. A análise dos erros cometidos por eles revela que, por não compreenderem os processos operatórios, aplicam as regras memorizadas sem refletirem sobre a validade ou não daquele conhecimento para a operação que irão realizar, o que sinaliza uma deficiência nos processos de ensino e de aprendizagem desse conteúdo.

Palavras-chave: Operações com Frações; Desempenho e Dificuldades; Educação Básica e Superior.

\begin{abstract}
The objective of this text is to discuss the performance and the difficulties presented by students in basic education and by undergraduates in the Teaching Degree Program in Mathematics in solving four fundamental operations with fractions. This discussion is based on the ideas by Llinares and Sánchez (1988), Monteiro and Groenwald (2014), and Silva and Almouloud (2008), among others. The data were
\end{abstract}

ReviSeM, Ano 2019, N. 2, p. $71-88$ 
collected by applying a questionnaire to 358 students in classes from the 7th and 9th years in elementary education, the 1st and 3rd years of secondary education, and undergraduates from Calculus I and II in the Teaching Degree Program in Mathematics. The results show that the performance of students in each one of the operations was unsatisfactory, despite showing improvement according to the academic level. An analysis of the errors they made reveals that, due to not understanding the order of operations, they apply memorized rules without reflecting on the validity or invalidity of that knowledge for the operation they are going to complete, which signals a deficiency in the teaching and learning processes of this content.

Keywords: Operations with Fractions; Performance and Difficulties; Basic and Higher Education.

\section{INTRODUÇÃO}

Este texto tem como propósito oportunizar uma discussão sobre o desempenho e as dificuldades apresentadas por estudantes da educação básica e por graduandos do curso de Licenciatura em Matemática na resolução das quatro operações fundamentais com frações. Os dados foram coletados em um projeto de pesquisa intitulado "Aprendizagem das operações com frações", desenvolvido no ano de 2018 como parte das atividades do Grupo de Estudo e Pesquisa em Educação Matemática (GEPEMAT).

Esta investigação dá continuidade ao projeto intitulado "Aritmética no Ensino Fundamental", desenvolvido por esse grupo de pesquisadores no ano de 2017, que tratou sobre os diferentes significados de fração. Nele, os dados foram coletados em turmas do $5^{\circ}$ ao $9^{\circ}$ ano do ensino fundamental e participaram 565 estudantes de quatro escolas de três municípios do interior do estado de Sergipe. Os resultados revelaram que o desempenho dos estudantes esteve abaixo de 50\% nos problemas relacionados aos significados: parte-todo, número, operador e medida, e que somente no problema que abordou o significado de quociente o desempenho esteve entre $40 \%$ e $60 \%$, o que sinaliza um despreparo desses alunos quanto aos diferentes significados de fração.

Esses resultados nos instigaram a buscar identificar as dificuldades apresentadas por estudantes de turmas de $7^{\circ}$ e $9^{\circ}$ ano do Ensino Fundamental, $1^{\circ}$ e $3^{\circ}$ ano do Ensino Médio, e $2^{\circ}$ período da graduação em Matemática - Licenciatura na resolução de operações fundamentais no conjunto $\mathrm{Q}$.

Assim, neste artigo fazemos uma discussão sobre os resultados relacionados à primeira questão do instrumento aplicado, a qual envolve oito operações com frações. Com vistas a fundamentar a discussão, delineia-se na sequência deste artigo um pequeno aporte teórico acerca do ensino e aprendizado das operações fundamentais com frações; a metodologia utilizada para coleta e análise dos dados; uma possibilidade de análise dos dados coletados; algumas reflexões e considerações, e as referências que embasaram o estudo. 


\section{APRENDIZADO DAS OPERAÇÕES COM FRAÇÕES}

O aprendizado dos números fracionários tem um histórico de bastante desagrado para professores e alunos quando se trata de sua compreensão, pois ainda se mantém uma elevada dificuldade de entender a fração como sendo um valor numérico, encarando-os muitas vezes até como meros símbolos.

As orientações dos Parâmetros Curriculares Nacionais para o ensino da matemática no segundo ciclo dos anos iniciais consideram o ensino das frações como fundamental para a aprendizagem dos números racionais. Para a construção do conceito de número racional, enfatizam a sua utilização em diferentes situações do cotidiano. Neste ciclo, os alunos devem ter contato com situações-problema cujas soluções não estejam no campo dos números naturais, possibilitando a aproximação destes ao conceito de número racional. Afirmam, ainda, que a representação decimal é percebida no cotidiano com mais frequência do que a forma fracionária e justificam essa ocorrência devido ao advento das calculadoras decimais (BRASIL, 1997).

Cavalieri (2005, p. 31) afirma que "o pouco uso das frações no cotidiano é uma das razões pelas quais as crianças sentem dificuldades com as frações, diariamente não são oferecidas oportunidades para que elas se familiarizem com essa ideia”. Realmente, quando o educador não busca trazer as situações cotidianas vivenciadas pelos alunos para a sala de aula, o aprendizado ocorre de forma isolada e, no caso deste conteúdo, os discentes não conseguem perceber as aplicações das frações no seu dia a dia.

Também, o pouco ou nenhum uso de materiais manipuláveis no ensino das operações com números racionais na forma de frações cria uma lacuna conceitual no entendimento do aluno (FREIRE e LIMA, 2019). Dessa forma, passa a aprender efetuando ações com símbolos que muitas vezes não têm significado conceitual para ele, o que resulta na memorização de um processo de regras.

As pesquisas revelam “[...] que mesmo alunos de nível médio ou superior apresentam dificuldades no trato com as frações e demonstram não conhecer aspectos relevantes do conceito de número racional, o que acarreta prejuízos à compreensão de novos conceitos matemáticos" (CAMPOS e RODRIGUES, 2007, p. 70).

De acordo com Lima e Brito (2005, p. 115), "para desenvolver corretamente o conceito de fração, a criança precisa ser solicitada a refletir sobre as seguintes questões: Qual é o todo? Quantos pedaços há no todo? São pedaços do mesmo tamanho?”. Acrescenta-se ainda que as crianças precisam, também, relacionar os nomes dessas partes, identificando-as como meios, 
terços, quartos, quintos, etc. A linguagem usada no tratamento desse conteúdo, a começar pela palavra fração e por termos como terços, quartos, quintos, não é uma linguagem natural das crianças nessa fase de escolaridade. O termo quarto pode, para eles, significar o lugar de dormir e terço um objeto usado na igreja para se fazer orações. Assim, segundo esses autores, também a linguagem matemática usada para comunicação em sala de aula traz complicadores.

Além das dificuldades destacadas, existem outras relacionadas aos diferentes significados de fração, pois a mesma pode ser entendida como: divisão, parte-todo, quantidade, equivalência, medida, entre outros. Por isso se torna um assunto complexo para o aprendizado de alunos que muitas vezes não sabem diferenciar corretamente a ideia presente na questão proposta pelo professor.

Llinares e Sánchez (1988) consideram que muitas vezes o estudante compreende o significado de fração em um tipo de situação e, nesse contexto, consegue representá-la na forma numérica ou com desenhos, entretanto, não consegue transferir esse conhecimento para outras situações, em contextos diversos, que envolvem outros significados.

De acordo com Campos, Silva e Pietropaolo (2009) uma forma de incentivar o aprendizado é propor atividades em contextos variados, que proporcionem aos estudantes realizarem com as frações atividades que desenvolvem com os Números Naturais, tal como somar, dividir e ordenar.

Muitas vezes o ensino das frações é dificultado por professores que somente conseguem associá-las às ideias de "fatias de pizza", "barras de chocolate", pois consideram que fora desse contexto é mais difícil encontrar situações que as contextualize. Assim, quando o educando se depara com situações fracionárias em outros contextos, não consegue identifica-las e nem operar com elas.

Lopes (2008) corrobora com essa ideia ao apontar em seus estudos algumas falhas ao ensinar frações que podem prejudicar muito a aprendizagem e a motivação dos alunos por tal tema. Dentre elas, o autor destaca a contextualização forçada, muitas vezes inadequada para alunos do ensino fundamental, o valor dado à nomenclatura inútil, e o ensino de regras e macetes para realizar as operações.

Nas escolas públicas brasileiras o ensino das operações com frações costuma acontecer no $5^{\circ}$ e no $6^{\circ}$ ano e, frequentemente, ocorre por meio do ensino das regras operatórias. Monteiro e Groenwald (2014) destacam que o mesmo deve iniciar com a resolução de problemas que envolvem frações com mesmos denominadores para depois trabalharem a soma de frações com denominadores diferentes. Ressaltam ainda que os estudantes do $7^{\circ}$ ano costumam fazer uso do 
menor múltiplo comum $(\mathrm{mmc})$ para resolver essas operações, entretanto, não dominam totalmente o procedimento.

No ponto de vista de Silva e Almouloud (2008), os alunos não costumam apresentar dificuldade na adição de números fracionários de mesmo denominador, por isso o foco passa a ser fazê-los compreender que quando os denominadores são diferentes, as frações têm nomes diferentes, ou seja, é como se o denominador identificasse a que "família" aquela fração pertence, por exemplo, a família dos meios, dos terços, dos quartos. Em decorrência disso, se faz necessário transformá-las em outras equivalentes a elas, que tenham o mesmo nome, isto é, o mesmo denominador. Os autores ressaltam que essa é a função do denominador, denominar, dar nome ao número de partes em que o inteiro foi dividido.

Para transformar frações em outras equivalentes e de mesmo denominador, esses autores reconhecem que o processo mais utilizado é o do menor múltiplo comum (mmc), contudo, acreditam "que tal procedimento prejudica a compreensão da definição da operação de adição" (SILVA e ALMOULOUD, 2008, p.61) e sugerem o produto de denominadores para transformar em frações equivalentes, pois esse processo oportuniza a compreensão das regras operatórias para essa operação.

Monteiro e Groenwald (2014), ao se referirem aos erros cometidos pelos estudantes ao somarem ou subtraírem frações, afirmam que o erro mais comum consiste na soma independente de numeradores e denominadores e consideram a possibilidade de que por já terem estudado o algoritmo da multiplicação de frações, possam estar aplicando essa ideia. Llinares e Sánchez (1988) explicam que esse tipo de erro pode ser causado pela semelhança que há entre os números Naturais e as frações, ou seja, os estudantes não veem a fração 4/7 eles veem o número 4 e o número 7 e operam com eles como se fossem independentes.

Para os pesquisadores, as causas dos erros cometidos pelos discentes ao operarem frações podem estar relacionadas à não compreensão do conceito, à elaboração de procedimentos pessoais alternativos aos que foram ensinados pelo professor, ao esquecimento de alguma etapa da regra ensinada. Também, ressaltam erros cometidos aleatoriamente, sinalizando desatenção, e erros que indicam que por não saber a resposta o estudante propõe um resultado qualquer. (LLINARES e SÁNCHEZ, 1988).

Com base nos aspectos destacados pelos autores citados, percebe-se que existem vários fatores que contribuem para o déficit no aprendizado das frações e que os estudos das dificuldades dos estudantes, embora represente somente uma visão momentânea da realidade, permite uma visão geral dos contextos investigados. 


\section{METODOLOGIA}

Este texto faz uso de abordagem qualitativa que assume uma perspectiva descritivaexplicativa ao comentar dados quantitativos, porque por meio dos dados coletados no instrumento aplicado aos estudantes da educação básica e ensino superior, buscamos compreender o contexto investigado, caracterizando-o e buscando apresentar indicativos que justifiquem as resoluções apresentadas pelos mesmos (FIORENTINI e LORENZATO, 2006).

Para identificar dificuldades apresentadas por estudantes da educação básica e da graduação em Matemática - Licenciatura na resolução de operações fundamentais no conjunto Q, aplicamos um questionário contendo cinco atividades: três exercícios e dois problemas envolvendo as operações de adição, subtração, multiplicação e divisão. Participaram da pesquisa discentes de turmas de $7^{\circ}$ e $9^{\circ}$ ano do Ensino Fundamental e de $1^{\circ}$ e $3^{\circ}$ ano do Ensino Médio de uma escola estadual do município de Itabaiana - Sergipe, escolhida por ter melhor desempenho na Prova Brasil. Optamos por começar pelo $7^{\circ}$ ano por considerarmos que no $6^{\circ}$ ano são ensinadas as operações com frações.

A aplicação do instrumento aos estudantes da Educação Básica ocorreu no dia 18 de junho de 2018, às 13h50min, para as turmas do turno vespertino e no dia 19 de junho de 2018, às 7h50min, para as turmas do turno matutino. A aplicação do instrumento aos estudantes da Licenciatura em Matemática ocorreu no dia 18 de julho de 2018 para a turma de Cálculo I e no dia 30 de julho de 2018 para a turma de Cálculo II, no horário de aula da disciplina. Participaram 358 estudantes, distribuídos conforme mostra o Quadro 1.

\begin{tabular}{|c|c|}
\hline Ano Escolar & Número de Alunos \\
\hline $7^{\circ}$ Ano Ensino Fundamental & 86 \\
\hline $9^{\circ}$ Ano Ensino Fundamental & 64 \\
\hline $1^{\circ}$ Ano Ensino Médio & 106 \\
\hline $3^{\circ}$ Ano Ensino Médio & 63 \\
\hline Cálculo I & 23 \\
\hline Cálculo II & 16 \\
\hline Total & $\mathbf{3 5 8}$ \\
\hline
\end{tabular}

Quadro 1 - Número de discentes por ano escolar Fonte: Banco de dados das autoras

A identificação dos protocolos dos estudantes aconteceu da seguinte forma: o protocolo “7A32" pertence ao estudante no 32 (32) da turma do sétimo ano A (7A), da Escola Básica; e nos protocolos dos graduandos registramos "CI2" para indicar que pertence ao graduando $\mathrm{n}^{\circ} 2$ (2) da turma de Cálculo I (CI) do Curso de Licenciatura em Matemática. 
A análise quantitativa buscou identificar a competência dos alunos na resolução das operações com frações. Levando em conta o desempenho dos estudantes como um reflexo da sua competência e considerando mais competente o aluno que mais acerta, fizemos uso da análise de acertos e erros. Para essa análise escolhemos categorizar as respostas em "corretas", "incorretas" e "em branco".

A análise qualitativa, além de buscar interpretar os dados quantitativos, voltou seu olhar para a produção escrita apresentada pelo discente, por considerarmos que ela revela evidências, instiga questionamentos e pode provocar mudanças na ação docente.

Cury (2008) afirma que as investigações apoiadas nos erros não têm o propósito de avaliar o aluno, mas de contribuir para compreender como ele se apropria de um determinado conhecimento e quais as dificuldades que ainda precisa superar até ser capaz de trabalhar com o conteúdo em questão.

\section{DISCUSSÃO SOBRE O DESEMPENHO E DIFICULDADES DOS ESTUDANTES}

Como destacado na metodologia, escolhemos apresentar e discutir neste texto alguns resultados sobre o desempenho dos estudantes na Questão 1 do instrumento. A Questão 1 aborda sobre as operações de adição, subtração, multiplicação e divisão de frações com denominadores iguais e diferentes e teve como propósito identificar se os estudantes sabem as regras básicas para operar com frações.

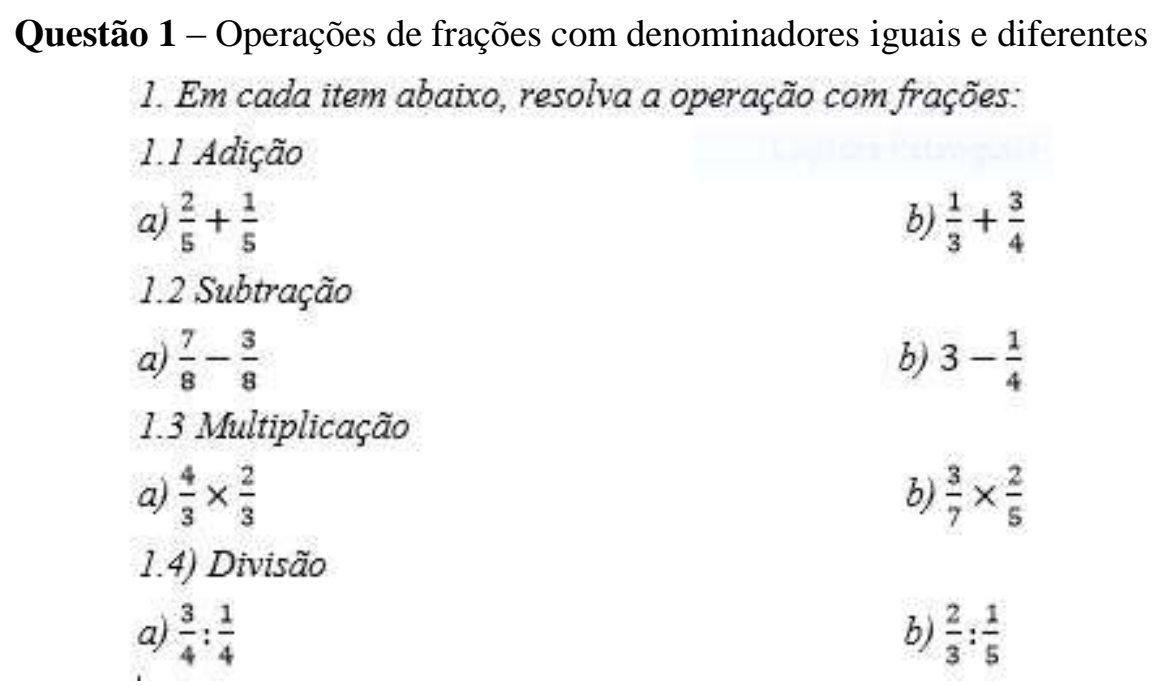

(Fonte: Banco de dados da pesquisa)

Optamos por analisar o desempenho em cada operação separadamente, e na sequência destacar os erros mais cometidos pelos estudantes em cada uma das operações por entendermos 
que os processos operatórios possuem características que os diferem. Iniciamos a discussão pela operação adição.

\section{Desempenho e dificuldades na operação adição}

Na Tabela 1 constam os resultados de desempenho, por nível de ensino, dos 358 estudantes na operação adição.

Tabela 1 - Desempenho geral dos estudantes na operação adição, por nível de ensino

\begin{tabular}{|c|c|c|c|c|c|c|c|c|c|c|c|c|}
\hline \multirow{2}{*}{ Adição } & \multicolumn{3}{|c|}{ Ensino Fundamental } & \multicolumn{4}{|c|}{ Ensino Médio } & \multicolumn{3}{c|}{ Ensino Superior } \\
\hline \multirow{2}{*}{ Item } & \multicolumn{2}{|c|}{$1.1 \mathrm{a}$} & \multicolumn{2}{|c|}{$1.1 \mathrm{~b}$} & \multicolumn{2}{|c|}{$1.1 \mathrm{a}$} & \multicolumn{2}{|c|}{$1.1 \mathrm{~b}$} & \multicolumn{2}{|c|}{$1.1 \mathrm{a}$} & \multicolumn{2}{|c|}{$1.1 \mathrm{~b}$} \\
\cline { 2 - 15 } & Freq. & $\%$ & Freq. & $\%$ & Freq. & $\%$ & Freq. & $\%$ & Freq. & $\%$ & Freq. & $\%$ \\
\hline $\mathrm{C}$ & 83 & $55 \%$ & 37 & $25 \%$ & 100 & $59 \%$ & 44 & $26 \%$ & 35 & $90 \%$ & 25 & $64 \%$ \\
\hline I & 67 & $45 \%$ & 112 & $75 \%$ & 67 & $40 \%$ & 123 & $73 \%$ & 4 & $10 \%$ & 13 & $33 \%$ \\
\hline EB & 0 & $0 \%$ & 1 & $1 \%$ & 2 & $1 \%$ & 2 & $1 \%$ & 0 & $0 \%$ & 1 & $3 \%$ \\
\hline Total & 150 & $100 \%$ & 150 & $100 \%$ & 169 & $100 \%$ & 169 & $100 \%$ & 39 & $100 \%$ & 39 & $100 \%$ \\
\hline
\end{tabular}

Fonte: Banco de dados das autoras

Os dados da Tabela 1 evidenciam que houve um crescimento no índice de acertos conforme o nível de escolaridade dos estudantes. No item "a", adição de frações com denominadores iguais, o desempenho esteve acima de $50 \%$ e variou de $55 \%$ no ensino fundamental a $90 \%$ no ensino superior. Observa-se que a diferença entre os índices do ensino fundamental e médio foi pequena (4\%). Nesse tipo de operação, era esperado um melhor desempenho dos estudantes da educação básica, considerando-se que os discentes não costumam apresentar dificuldade na soma de frações com mesmo denominador (SILVA e ALMOULOUD, 2008). No item “b”, adição de frações com denominadores diferentes, o desempenho não chegou a 30\% nas turmas da educação básica e não chegou a $70 \%$ no ensino superior, evidenciando dificuldade nesse tipo de situação.

Essa diferença nos levou a buscar observar o desempenho de cada aluno nos itens "a e b", em cada nível de ensino. Para tanto, organizamos tabelas que representam matrizes quadradas de ordem três que nos mostram o percentual de estudantes que acertou os dois itens $\left(a_{11}\right)$; que certou o item "a" e errou o "b" $\left(a_{12}\right)$; que acertou o item "a" e deixou em branco o "b" (a13); e assim sucessivamente. 
Tabela 2 - Desempenho dos alunos do ensino fundamental nos itens "a e b" da adição

\begin{tabular}{|c|c|c|c|c|}
\hline \multicolumn{5}{|c|}{ Ensino Fundamental } \\
\hline $\begin{array}{c}\text { 1.1 } \\
1.1 \mathrm{~b}\end{array}$ & $\mathrm{C}$ & $\mathrm{I}$ & $\mathrm{EB}$ & Total \\
\hline $\mathrm{C}$ & 35 & 47 & 1 & 83 \\
\hline I & 2 & 65 & 0 & 67 \\
\hline EB & 0 & 0 & 0 & 0 \\
\hline Total & 37 & 112 & 1 & 150 \\
\hline
\end{tabular}

Fonte: Banco de dados das autoras
Tabela 3 - Desempenho dos alunos do ensino médio nos itens "a e b" da adição

\begin{tabular}{|c|c|c|c|c|}
\hline \multicolumn{5}{|c|}{ Ensino Médio } \\
\hline $\begin{array}{c}\text { 1.1. b } \\
1.1 \mathrm{a}\end{array}$ & $\mathrm{C}$ & $\mathrm{I}$ & $\mathrm{EB}$ & Total \\
\hline $\mathrm{C}$ & 43 & 57 & 0 & 100 \\
\hline $\mathrm{I}$ & 1 & 66 & 0 & 67 \\
\hline EB & 0 & 0 & 2 & 2 \\
\hline Total & 44 & 123 & 2 & 169 \\
\hline
\end{tabular}

Fonte: Banco de dados das autoras
Tabela 4 - Desempenho dos alunos do ensino superior nos itens "a e b" da adição

\begin{tabular}{|c|c|c|c|c|}
\hline \multicolumn{5}{|c|}{ Ensino Superior } \\
\hline $\begin{array}{c}\text { 1.1. } \\
\text { 1.1 }\end{array}$ & C & I & EB & Total \\
\hline C & 24 & 10 & 1 & 35 \\
\hline I & 1 & 3 & 0 & 4 \\
\hline EB & 0 & 0 & 0 & 0 \\
\hline Total & 25 & 13 & 1 & 39 \\
\hline
\end{tabular}

Fonte: Banco de dados das autoras

As Tabelas 2 e 3 nos permitem perceber que na educação básica um maior número de estudantes errou os dois itens: 65 no ensino fundamental (43\%) e 66 no ensino médio (39\%), o que evidencia o despreparo dos mesmos na resolução desse tipo de operação. Também, observa-se que no ensino fundamental $47(31 \%)$ e no médio 57 (34\%) acertaram o item "a" e erram o item "b", sinalizando que a diferença nos procedimentos operatórios para realizar a adição de frações com denominadores iguais ou diferentes influencia no desempenho dos alunos. Ainda, em menor número, destaca-se a quantidade de estudantes que acertou os dois itens, 35 (23\%) no fundamental e 43 (25\%) no médio. Os índices de percentual nos mostram que entre esses níveis de ensino as diferenças percentuais não são significativas.

No ensino superior a maioria dos estudantes das disciplinas de Cálculo I e II acertou os dois itens (62\%), demonstrando melhor desempenho, contudo, ficaram abaixo do esperado, pois principalmente na turma de Cálculo II é de se esperar que 100\% da turma domine a resolução desse tipo de operação.

Consideramos importante ressaltar que o erro mais cometido por esses discentes ao realizarem a adição de frações foi somar numerador com numerador e denominador com denominador, conforme ilustrado na Figura 1.
1.1 Adiçăo
a) $\frac{2}{5}+\frac{1}{5}=\frac{3}{10}$
b) $\frac{1}{3}+\frac{3}{4} \quad \frac{4}{7}$

Figura 1- Protocolo do Estudante 3B10

Fonte: Banco de dados das autoras

$\mathrm{Na}$ solução apresentada pelo estudante da turma B do terceiro ano do ensino médio (3B10) observamos que ele somou numerador com numerador e denominador com 
denominador, fazendo uma analogia à regra da multiplicação de frações, conforme constatado por Monteiro e Groenwald (2014). Esse tipo de erro foi identificado em 198 protocolos, o que corresponde a $55 \%$ do total de 358 estudantes que participaram da pesquisa. Escolhemos apresentar o protocolo de um aluno do ensino médio porque foi nesse nível de ensino que esse erro apareceu mais vezes, dos 169 estudantes do ensino médio, 123 erraram o item "b"; e desses, $101(60 \%)$ cometeram esse tipo de erro.

\section{Desempenho e dificuldades na operação subtração}

Na Tabela 5 constam os resultados de desempenho, por nível de ensino, na operação subtração.

Tabela 5 - Desempenho geral dos estudantes na operação subtração, por nível de ensino

\begin{tabular}{|c|c|c|c|c|c|c|c|c|c|c|c|c|}
\hline Subtração & \multicolumn{3}{|c|}{ Ensino Fundamental } & \multicolumn{4}{|c|}{ Ensino Médio } & \multicolumn{3}{c|}{ Ensino Superior } \\
\hline \multirow{2}{*}{ Item } & \multicolumn{2}{|c|}{$1.2 \mathrm{a}$} & \multicolumn{2}{|c|}{$1.2 \mathrm{~b}$} & \multicolumn{2}{|c|}{$1.2 \mathrm{a}$} & \multicolumn{2}{|c|}{$1.2 \mathrm{~b}$} & \multicolumn{2}{|c|}{$1.2 \mathrm{a}$} & \multicolumn{2}{|c|}{$1.2 \mathrm{~b}$} \\
\cline { 2 - 15 } & Freq. & $\%$ & Freq. & $\%$ & Freq. & $\%$ & Freq. & $\%$ & Freq. & $\%$ & Freq. & $\%$ \\
\hline $\mathrm{C}$ & 70 & $47 \%$ & 23 & $15 \%$ & 101 & $60 \%$ & 41 & $24 \%$ & 32 & $82 \%$ & 21 & $54 \%$ \\
\hline $\mathrm{I}$ & 80 & $53 \%$ & 120 & $80 \%$ & 63 & $37 \%$ & 115 & $68 \%$ & 7 & $18 \%$ & 16 & $41 \%$ \\
\hline EB & 0 & $0 \%$ & 7 & $5 \%$ & 5 & $3 \%$ & 13 & $8 \%$ & 0 & $0 \%$ & 2 & $5 \%$ \\
\hline Total & 150 & $100 \%$ & 150 & $100 \%$ & 169 & $100 \%$ & 169 & $100 \%$ & 39 & $100 \%$ & 39 & $100 \%$ \\
\hline
\end{tabular}

Fonte: Banco de dados das autoras

Observando-se os índices percentuais de cada item na Tabela 5, percebe-se que o desempenho geral dos estudantes foi crescente, conforme o avanço no nível de ensino. No item "a" partiu de 47\% no ensino fundamental, teve um avanço no ensino médio (60\%) e chegou a $82 \%$ no ensino superior. No item "b" partiu de $15 \%$ no ensino fundamental, teve um avanço no ensino médio (24\%) e chegou a $54 \%$ no ensino superior. Como não são os mesmos estudantes nos diferentes níveis de ensino, não se pode afirmar que esses dados mostram um avanço no aprendizado deles, contudo, esse resultado indica o aumento no amadurecimento educacional dos discentes.

Vale destacar que apesar de que a resolução das operações de adição e a subtração de frações fazer uso da mesma regra operatória, em todos os níveis de ensino, o índice de desempenho foi menor na operação subtração.

Da mesma forma que na adição, buscamos olhar de maneira mais detalhada para verificar o desempenho dos estudantes em cada item, conforme mostra nas tabelas 6, 7 e 8 . 
Tabela 6 - Desempenho dos alunos do ensino fundamental nos itens "a e b" da subtração

\begin{tabular}{|c|c|c|c|c|}
\hline \multicolumn{5}{|c|}{ Ensino Fundamental } \\
\hline $\begin{array}{c}1.2 \mathrm{a} \\
\text { 1.2b }\end{array}$ & $\mathrm{C}$ & $\mathrm{I}$ & $\mathrm{EB}$ & Total \\
\hline $\mathrm{C}$ & 20 & 48 & 2 & 70 \\
\hline $\mathrm{I}$ & 3 & 72 & 5 & 80 \\
\hline EB & 0 & 0 & 0 & 0 \\
\hline Total & 23 & 120 & 7 & 150 \\
\hline
\end{tabular}

Fonte: Banco de dados das autoras
Tabela 7 - Desempenho dos alunos do ensino médio nos itens "a e b" da subtração

\begin{tabular}{|c|c|c|c|c|}
\hline \multicolumn{5}{|c|}{ Ensino Médio } \\
\hline $\begin{array}{c}1.2 \mathrm{a} \\
1.2 \mathrm{a}\end{array}$ & $\mathrm{C}$ & $\mathrm{I}$ & $\mathrm{EB}$ & Total \\
\hline $\mathrm{C}$ & 40 & 59 & 2 & 101 \\
\hline $\mathrm{I}$ & 1 & 56 & 6 & 63 \\
\hline EB & 0 & 0 & 5 & 5 \\
\hline Total & 41 & 115 & 13 & 169 \\
\hline
\end{tabular}

Fonte: Banco de dados das autoras
Tabela 8 - Desempenho dos alunos do ensino superior nos itens "a e b" da subtração

\begin{tabular}{|c|c|c|c|c|}
\hline \multicolumn{5}{|c|}{ Ensino Superior } \\
\hline $\begin{array}{c}1.2 \mathrm{a} \\
1.2 \mathrm{~b}\end{array}$ & $\mathrm{C}$ & $\mathrm{I}$ & $\mathrm{EB}$ & Total \\
\hline $\mathrm{C}$ & 18 & 12 & 2 & 32 \\
\hline $\mathrm{I}$ & 3 & 4 & 0 & 7 \\
\hline EB & 0 & 0 & 0 & 0 \\
\hline Total & 21 & 16 & 2 & 39 \\
\hline
\end{tabular}

Fonte: Banco de dados das autoras

Ao comparar o desempenho dos estudantes nos itens "a" e "b" da subtração, observa-se que no ensino fundamental o número de estudantes que subtraiu corretamente quando as frações têm o mesmo denominador e errou quando os denominadores são diferentes (32\%) é mais do que o dobro dos que acertaram os dois casos (13\%). No ensino médio, embora a diferença não seja tão grande, também o maior número acertou o item "a" e errou o item "b". Esse resultado indica uma dificuldade na operação de frações com denominadores diferentes. No ensino superior, essa diferença não ocorreu, entretanto, menos da metade dos estudantes (46\%) acertou os dois itens.

As respostas escritas dos estudantes nos mostram que a estratégia incorreta mais utilizada foi a de subtrair numerador com numerador e denominador com denominador.
1.2 Subtração
a) $\frac{7}{8}-\frac{3}{8}=\frac{4}{0}$
b) $3-\frac{1}{4}=\frac{2}{3}$

Figura 2 - Protocolo do Estudante 1B13

Fonte: Banco de dados das autoras

A Figura 2 destaca a estratégia utilizada por um estudante do $1^{\circ}$ ano do ensino médio. Observa-se que no item "a" ele subtraiu os numeradores $(7-3)$ e os denominadores $(8-8)$ e no item "b" seguiu o mesmo raciocínio para os numeradores, subtraindo 3 - 1, contudo, como o denominador 1 é menor do que 4, fez 4 - 1. Erros envolvendo a ideia de subtração de numeradores e denominadores foram encontrados nos protocolos de 133 estudantes. Destes, 69 são do ensino fundamental e representam $46 \%$ do total de estudantes desse nível de ensino; 59 são do ensino médio e representam 35\% do total de estudantes desse nível de ensino. Em menor 
número, pois foram somente 5 estudantes, o que corresponde a $13 \%$ do total de estudantes desse nível de ensino, esse erro foi identificado nos protocolos do ensino superior.

Ainda, destaca-se que 44 estudantes do ensino médio, o que corresponde a $26 \%$ desse grupo de alunos, erraram porque para realizar a subtração das frações, subtraíram os numeradores e copiaram um dos denominadores. Na Figura 2 destacamos a solução apresentada por um estudante da turma $\mathrm{C}$ do terceiro ano do ensino médio (3C12).

$$
\begin{aligned}
& \text { 1.2 Subtração } \\
& \begin{array}{ll}
\text { a) } \frac{7}{8}-\frac{3}{8} \frac{4}{8} & \text { b) } 3-\frac{1}{4} \frac{2}{4}
\end{array}
\end{aligned}
$$

Figura 3 - Protocolo do Estudante 3C12

Fonte: Banco de dados das autoras

A representação escrita apresentada pelo estudante mostra que ele acertou a operação proposta no item "a" ao subtrair os numeradores e conservar o denominador, mas errou o item “b” porque aplicou o mesmo raciocínio e, nesse caso, esse procedimento não é válido porque estamos subtraindo frações com denominadores diferentes.

\section{Desempenho e dificuldades na operação multiplicação}

Na Tabela 9 constam os resultados de desempenho por nível de ensino na operação multiplicação.

Tabela 9 - Desempenho geral dos estudantes na operação multiplicação, por nível de ensino

\begin{tabular}{|c|c|c|c|c|c|c|c|c|c|c|c|c|}
\hline Multiplicação & \multicolumn{3}{|c|}{ Ensino Fundamental } & \multicolumn{4}{|c|}{ Ensino Médio } & \multicolumn{3}{c|}{ Ensino Superior } \\
\hline \multirow{2}{*}{ Item } & \multicolumn{2}{|c|}{$1.3 \mathrm{a}$} & \multicolumn{2}{|c|}{$1.3 \mathrm{~b}$} & \multicolumn{2}{|c|}{$1.3^{\mathrm{a}}$} & \multicolumn{2}{|c|}{$1.3 \mathrm{~b}$} & \multicolumn{2}{|c|}{$1.3 \mathrm{a}$} & \multicolumn{2}{c|}{$1.3 \mathrm{~b}$} \\
\cline { 2 - 16 } & Freq. & $\%$ & Freq. & $\%$ & Freq. & $\%$ & Freq. & $\%$ & Freq. & $\%$ & Freq. & $\%$ \\
\hline $\mathrm{C}$ & 58 & $39 \%$ & 59 & $39 \%$ & 53 & $31 \%$ & 72 & $43 \%$ & 24 & $62 \%$ & 24 & $62 \%$ \\
\hline I & 91 & $61 \%$ & 90 & $60 \%$ & 113 & $67 \%$ & 91 & $54 \%$ & 15 & $38 \%$ & 13 & $33 \%$ \\
\hline EB & 1 & $1 \%$ & 1 & $1 \%$ & 3 & $2 \%$ & 6 & $4 \%$ & 0 & $0 \%$ & 2 & $5 \%$ \\
\hline Total & 150 & $100 \%$ & 150 & $100 \%$ & 169 & $100 \%$ & 169 & $100 \%$ & 39 & $100 \%$ & 39 & $100 \%$ \\
\hline
\end{tabular}

Fonte: Banco de dados das autoras

Assim como nas operações de adição e subtração, também na multiplicação houve aumento no índice de desempenho conforme avançou o nível de ensino. No entanto, por observarmos que o erro mais cometido na adição foi a soma de numerador com numerador e denominador com denominador, o que nos sinaliza uma aplicação inadequada da regra operatória da multiplicação na adição de frações, conforme citado por Monteiro e Groenwald 
(2014), esperávamos que o desempenho nessa operação fosse maior do que nas outras, contudo, a Tabela 9 nos mostra que isso não aconteceu no ensino fundamental e médio, pois os índices ficaram abaixo de $45 \%$ e nem no ensino superior, que teve somente $62 \%$ de acertos em cada item.

Diferentemente das operações de adição e subtração, na multiplicação não houve grande diferença entre o número de acertos nos itens "a" e "b". As tabelas 10, 11 e 12 nos mostram a relação de desempenho dos estudantes entre os itens.

Tabela 10 - Desempenho dos alunos do ensino fundamental nos itens "a e b" da multiplicação

\begin{tabular}{|c|c|c|c|c|}
\hline \multicolumn{5}{|c|}{ Ensino Fundamental } \\
\hline $1.3 \mathrm{a}$ & $\mathrm{C}$ & $\mathrm{I}$ & $\mathrm{EB}$ & Total \\
\hline $\mathrm{C}$ & 49 & 9 & 0 & 58 \\
\hline $\mathrm{I}$ & 10 & 81 & 0 & 91 \\
\hline EB & 0 & 0 & 1 & 1 \\
\hline Total & 59 & 90 & 1 & 150 \\
\hline
\end{tabular}

Fonte: Banco de dados das autoras
Tabela 11 - Desempenho dos alunos do ensino médio nos itens "a e b" da multiplicação

\begin{tabular}{|c|c|c|c|c|}
\hline \multicolumn{5}{|c|}{ Ensino Médio } \\
\hline $\begin{array}{c}1.3 \mathrm{a} \\
\text { 1.3b }\end{array}$ & $\mathrm{C}$ & $\mathrm{I}$ & $\mathrm{EB}$ & Total \\
\hline $\mathrm{C}$ & 47 & 6 & 0 & 53 \\
\hline $\mathrm{I}$ & 25 & 85 & 3 & 113 \\
\hline $\mathrm{EB}$ & 0 & 0 & 3 & 3 \\
\hline Total & 72 & 91 & 6 & 169 \\
\hline
\end{tabular}

Fonte: Banco de dados das autoras
Tabela 12 - Desempenho dos alunos do ensino superior nos itens "a e b" da multiplicação

\begin{tabular}{|c|c|c|c|c|}
\hline \multicolumn{5}{|c|}{ Ensino Superior } \\
\hline $1.3 \mathrm{a}$ & $\mathrm{C}$ & $\mathrm{I}$ & $\mathrm{EB}$ & Total \\
\hline $\mathrm{C}$ & 22 & 2 & 0 & 24 \\
\hline $\mathrm{I}$ & 2 & 11 & 2 & 15 \\
\hline EB & 0 & 0 & 0 & 0 \\
\hline Total & 24 & 13 & 2 & 39 \\
\hline
\end{tabular}

Fonte: Banco de dados das autoras

As tabelas evidenciam que em todos os níveis de ensino a maioria dos estudantes acertou os dois itens relacionados a multiplicação de frações, o que já era esperado porque a regra operatória é a mesma. Diferentemente do que aconteceu na adição e na subtração, cujos resultados mostraram um menor desempenho quando as frações têm denominadores diferentes, em virtude de não haver diferença na regra operatória para a solução de cada item, os resultados mostram que tanto no ensino fundamental quanto no médio é maior o número de estudantes que errou a multiplicação de frações com denominadores iguais e acertou a multiplicação de frações com denominadores diferentes.

\begin{tabular}{ll}
\hline 1.3 Multiplicação & b) $\frac{3}{7} \times \frac{2}{5}=\frac{6}{35}$ \\
a) $\frac{4}{3} \times \frac{2}{3}=\frac{8}{3}$ &
\end{tabular}

Figura 4 - Protocolo do Estudante 7B28

Fonte: Banco de dados das autoras

Esse estudante do $7^{\circ}$ ano B realizou corretamente a operação proposta no item "b", multiplicando numerador por numerador e denominador por denominador, no entanto, no item 
"a" multiplicou os numeradores e conservou o denominador, fazendo uma analogia ao processo aditivo de frações com mesmo denominador.

Além desse tipo de erro, as respostas escritas dos estudantes nos mostram que a estratégia incorreta mais utilizada foi a de multiplicar o numerador de uma fração com o denominador da outra fração (multiplicação cruzada), conforme pode se verificar nas soluções dadas pelo estudante de Cálculo II.

\begin{tabular}{ll}
1.3 Multiplicação & b) $\frac{3}{7} \times \frac{2}{5}=\frac{15}{14}$ \\
a) $\frac{4}{3} \times \frac{2}{3}=\frac{12}{6}=2$ & \\
\hline
\end{tabular}

Figura 5 - Protocolo do Estudante CII8

Fonte: Banco de dados das autoras

Entende-se que esse aluno vê a multiplicação de frações como uma igualdade de razões e, por isso, aplica a propriedade da proporção que afirma que o produto dos meios é igual ao produto dos extremos. Ao todo, foram 137 estudantes que cometeram esse erro: 64 (43\%) do ensino fundamental; 63 (37\%) do ensino médio; e 10 (26\%) do ensino superior.

\section{Desempenho e dificuldades na operação divisão}

Na Tabela 13 constam os resultados de desempenho, por nível de ensino, na operação divisão.

Tabela 13 - Desempenho geral dos estudantes na operação divisão, por nível de ensino

\begin{tabular}{|c|c|c|c|c|c|c|c|c|c|c|c|c|}
\hline Divisão & \multicolumn{3}{|c|}{ Ensino Fundamental } & \multicolumn{4}{c|}{ Ensino Médio } & \multicolumn{3}{c|}{ Ensino Superior } \\
\hline \multirow{2}{*}{ Item } & \multicolumn{2}{|c|}{$1.4 \mathrm{a}$} & \multicolumn{2}{|c|}{$1.4 \mathrm{~b}$} & \multicolumn{2}{|c|}{$1.4 \mathrm{a}$} & \multicolumn{2}{|c|}{$1.4 \mathrm{~b}$} & \multicolumn{3}{|c|}{$1.4 \mathrm{a}$} & \multicolumn{1}{|c|}{$1.4 \mathrm{~b}$} \\
\cline { 2 - 15 } & Freq. & $\%$ & Freq. & $\%$ & Freq. & $\%$ & Freq. & $\%$ & Freq. & $\%$ & Freq. & $\%$ \\
\hline C & 66 & $44 \%$ & 37 & $25 \%$ & 75 & $44 \%$ & 47 & $28 \%$ & 21 & $54 \%$ & 18 & $46 \%$ \\
\hline I & 72 & $48 \%$ & 98 & $65 \%$ & 81 & $48 \%$ & 98 & $58 \%$ & 16 & $41 \%$ & 17 & $44 \%$ \\
\hline EB & 12 & $8 \%$ & 15 & $10 \%$ & 13 & $8 \%$ & 24 & $14 \%$ & 2 & $5 \%$ & 4 & $10 \%$ \\
\hline Total & 150 & $100 \%$ & 150 & $100 \%$ & 169 & $100 \%$ & 169 & $100 \%$ & 39 & $100 \%$ & 39 & $100 \%$ \\
\hline
\end{tabular}

Fonte: Banco de dados das autoras

Os dados da Tabela 13 nos mostram que no item "a" o percentual de desempenho dos estudantes da educação básica foi igual (44\%) e que no ensino superior foi um pouco maior (54\%). No item "b" o percentual variou de $25 \%$ no ensino fundamental, passando por $28 \%$ no ensino médio e chegando a $46 \%$ no ensino superior. É um desempenho baixo, contudo já era 
esperado, pois essa operação apresenta dificuldade para os estudantes, mesmo quando o cálculo envolve números naturais.

As tabelas 14, 15 e 16 nos mostram a relação de desempenho dos estudantes entre os itens "a" e "b".

Tabela 14 - Desempenho dos alunos do ensino fundamental nos itens "a e b" da divisão

\begin{tabular}{|c|c|c|c|c|}
\hline \multicolumn{5}{|c|}{ Ensino Fundamental } \\
\hline $\begin{array}{l}1.4 \mathrm{~b} \\
1.4 \mathrm{a}\end{array}$ & $\mathrm{C}$ & I & EB & Total \\
\hline $\mathrm{C}$ & 35 & 30 & 1 & 66 \\
\hline I & 2 & 67 & 3 & 72 \\
\hline EB & 0 & 1 & 11 & 12 \\
\hline Total & 37 & 98 & 15 & 150 \\
\hline
\end{tabular}

Fonte: Banco de dados das autoras
Tabela 15 - Desempenho dos alunos do ensino médio nos itens "a e b" da divisão

\begin{tabular}{|c|c|c|c|c|}
\hline \multicolumn{5}{|c|}{ Ensino Médio } \\
\hline $\begin{array}{c}1.4 \mathrm{a} \\
\mathrm{C}\end{array}$ & $\mathrm{C}$ & $\mathrm{I}$ & $\mathrm{EB}$ & Total \\
\hline $\mathrm{I}$ & 70 & 28 & 7 & 75 \\
\hline EB & 0 & 1 & 12 & 13 \\
\hline Total & 47 & 98 & 24 & 169 \\
\hline
\end{tabular}

Fonte: Banco de dados das autoras
Tabela 16 - Desempenho dos alunos do ensino superior nos itens "a e b" da divisão

\begin{tabular}{|c|c|c|c|c|}
\hline \multicolumn{5}{|c|}{ Ensino Superior } \\
\hline $1.4 \mathrm{a}$ & $\mathrm{C}$ & $\mathrm{I}$ & $\mathrm{EB}$ & Total \\
\hline $\mathrm{C}$ & 17 & 3 & 1 & 21 \\
\hline $\mathrm{I}$ & 1 & 13 & 2 & 16 \\
\hline $\mathrm{EB}$ & 0 & 1 & 1 & 2 \\
\hline Total & 18 & 17 & 4 & 39 \\
\hline
\end{tabular}

Fonte: Banco de dados das autoras

Os resultados presentes nas tabelas 14, 15 e 16 deixam claro que aumentou o número de estudantes da educação básica que deixou em branco os dois itens. E, também, que nos três níveis de ensino, aumentou o número de estudantes que errou os dois itens. Esse resultado vai ao encontro da afirmação feita por Silva e Almouloud (2008) de que é na operação divisão que os alunos apresentam mais dificuldade, mesmo quando lidam com números naturais.

O percentual de alunos que acertou os dois itens foi de $23 \%$ no ensino fundamental, $24 \%$ no ensino médio, e $44 \%$ no ensino superior. Esses dados mostram que o índice de desempenho dos estudantes do ensino fundamental foi semelhante ao do ensino médio.

O percentual de estudantes que acertou a divisão de frações com denominadores iguais e errou a divisão com denominadores diferentes foi de $20 \%$ no ensino fundamental, $17 \%$ no ensino médio, e $8 \%$ no ensino superior. Ao que tudo indica, apesar da regra operatória da divisão ser a mesma para os dois casos, os estudantes não têm essa percepção.

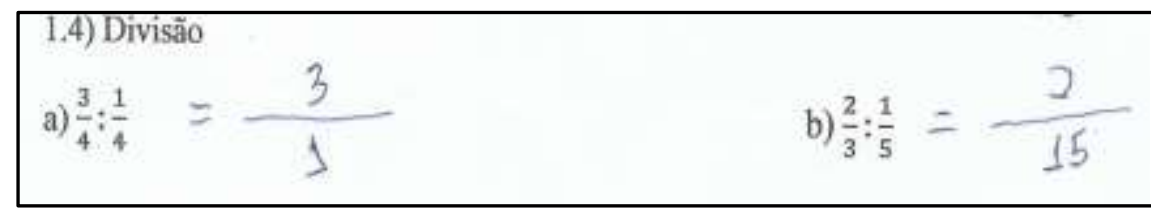

Figura 6 - Protocolo do Estudante 7A21

Fonte: Banco de dados das autoras

O estudante do $7^{\circ}$ Ano A apresentou como resolução somente o registro do resultado final da operação que realizou, sem deixar claro qual procedimento utilizou. É possível que no 
item "a" ele tenha dividido numerador por numerador $(3 \div 1)$ e denominador por denominador $(4 \div 4)$, contudo, no item "b", talvez porque a divisão dos denominadores não é exata, ele multiplicou.

Ainda, identificamos que um grupo de estudantes ao dividir as frações dividiu numerador por numerador e copiou um dos denominadores, conforme mostram as soluções dadas pelo estudante do $7^{\circ}$ ano A.

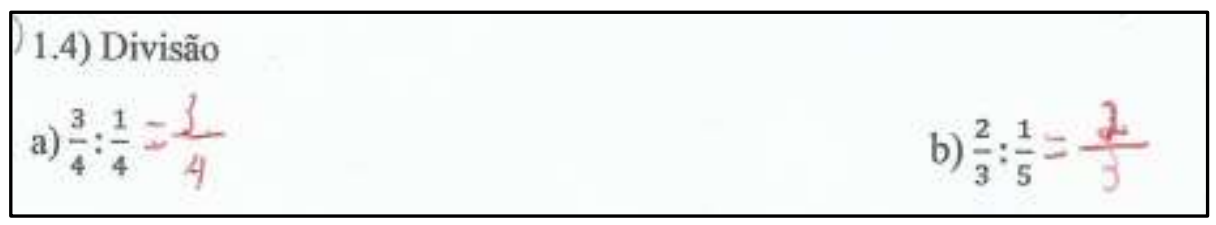

Figura 7 - Protocolo do Estudante 7A26

Fonte: Banco de dados das autoras

No item "a" a resposta dada pelo aluno nos levou a pensar que ele dividiu 3 por 1 e conservou o 4 como denominador, fazendo uma analogia com a regra da adição de frações com mesmo denominador. Contudo, o que causa preocupação é que ao verificar no protocolo desse estudante como ele realizou a soma de frações com mesmo denominador, percebeu-se que ele errou, pois somou numerador com numerador e denominador com denominador. Essa constatação nos indica que ele conhece algumas regras operatórias de frações, porém, não sabe para qual operação ela tem validade.

No item "b" esse estudante dividiu 2 por 1 e escolheu o 3 para denominador. Ao todo, foram 74 estudantes que cometeram esse erro: 20 (13\%) do ensino fundamental; 45 (27\%) do ensino médio; e 9 (23\%) do ensino superior. Observa-se que, em comparação com as outras operações, esses índices estão baixos, isso se deve ao fato de que foi na operação divisão que ocorreu uma variação maior nos tipos de erros. Foram eles: escolheu uma das frações como resposta (22), posicionou errado o resultado da multiplicação cruzada (15), multiplicou numerador com numerador e denominador com denominador (12), errou ao realizar o cálculo (13), usou o m.m.c. (9). Esses erros revelam que os estudantes buscam aplicar qualquer conhecimento relacionado às frações, sem se questionarem se aquele conhecimento é válido para a situação em questão, no caso, a divisão de frações.

Da mesma forma que Llinares e Sánchez (1988), encontramos erros nos quais o estudante por não saber a resposta correta, propôs um resultado ao acaso. Na operação divisão 
isso se deu em 78 respostas, e por não conseguirmos entender o que o estudante fez, consideramos como "erro não identificado".

\section{CONSIDERAÇÕES FINAIS}

A discussão, presente neste texto, sobre o desempenho e as dificuldades apresentadas por estudantes da educação básica e por graduandos do curso de Licenciatura em Matemática na resolução das quatro operações fundamentais com frações, mesmo que limitada ao contexto investigado, busca corroborar na compreensão de aspectos que limitam o aprendizado dos estudantes, tais como, a memorização de regras, sem o entendimento do que significam.

Os resultados mostram que o índice de desempenho dos estudantes em cada uma das operações foi insatisfatório, apesar de evidenciar crescimento conforme o nível de escolaridade. A análise dos erros cometidos pelos estudantes revela que por não compreenderem os processos operatórios, os estudantes aplicam as regras memorizadas em qualquer contexto, sem refletirem sobre a validade ou não daquele conhecimento para a operação que irá realizar, o que sinaliza uma deficiência nos processos de ensino e de aprendizagem desse conteúdo.

Os resultados reforçam a ideia de que o ensino das operações com números racionais deve ser repensado, pois não está oportunizando a compreensão das regras operatórias com significado. Nesse sentido, sugere-se a realização de uma proposta de formação continuada com professores do ensino fundamental para que a discussão desses resultados possibilite que revejam sua forma de ensinar e consigam propor atividades que oportunizem aos estudantes a construção de conhecimentos iniciais bem fundamentados, o que lhes permitirá melhor desempenho tanto nas operações com frações como em outros conhecimentos matemáticos.

\section{REFERÊNCIAS}

BRASIL. Parâmetros Curriculares Nacionais: Matemática. Brasília: MEC/SEF, 1997.

CAVALIERI, L. O Ensino das Frações. (Monografia da especialização em Ensino de Matemática). Umuarama - PR, Universidade Paranaense, 2005.

CAMPOS, T. M. M., SILVA, A. F. G.; PIETROPAOLO, R. C. Considerações a respeito do ensino e aprendizagem de representações fracionárias de números racionais. In:

GUIMARÃES, G. B.; ROSA, R. E. S. (Org.). Reflexões sobre o ensino de matemática nos anos iniciais de escolarização. Recife: SBEM, p. 131-139, 2009.

CAMPOS, T. M. M.; RODRIGUES, W. R. A ideia de unidade na construção do conceito do número racional. Revemat: Revista Eletrônica de Educação Matemática. Florianópolis (SC), 
v.2, n.4, p. 68-93, 2007. Disponível em

https://periodicos.ufsc.br/index.php/revemat/article/download/12992/12093

CURY, H. N. Análise de erros: o que podemos aprender com as respostas dos alunos. Belo Horizonte: Autêntica, 2008.

FIORENTINI, D.; LORENZATO, S. Investigação em Educação Matemática: percursos teóricos e metodológicos. Campinas, SP: Autores Associados, 2006.

FREIRE, P. C.; LIMA, R. N. Números racionais na forma fracionária: a influência de características formais. Revisem. Itabaiana (SE), v.4, n. 1, p.37-51, 2019. Disponível em https://seer.ufs.br/index.php/ReviSe/issue/view/801/showToc

LLINARES, S.; SÁNCHEZ, M. V. Fracciones: la relacion parte-todo. Madrid: Sintesis, 1988.

LIMA, V. S.; BRITO, R. F. de. Mapeamento cognitivo e a formação do conceito de frações. In: BRITO, R. F. (Org.). Psicologia da Educação Matemática: teoria e pesquisa.

Florianópolis, SC: Insular, 2005.

LOPES, A. J. O que nossos alunos podem estar deixando de aprender sobre frações, quando tentamos lhes ensinar frações. Bolema, Rio Claro (SP), v.21, n. 31, p.1-22, 2008. Disponível em

https://www.researchgate.net/publication/277786999_O_Que_Nossos_Alunos_Podem_Estar_ Deixando_de_Aprender_Sobre_Fracoes_Quando_Tentamos_lhes_Ensinar_Fracoes

MONTEIRO, A. B.; GROENWALD, C. L. O. Dificuldades na aprendizagem de frações: reflexões a partir de uma experiência utilizando testes adaptativos. Alexandria: Revista de Educação em Ciência e Tecnologia, v. 7, n. 2, p. 103-135, 2014. Disponível em https://periodicos.ufsc.br/index.php/alexandria/article/download/38217/29121

SILVA, M. J. F.; ALMOULOUD, S. As operações com números racionais e seus significados a partir da concepção parte-todo. Bolema, Rio Claro (SP), Ano 21, n. 31, p. 55-78, 2008.

Disponível em https://www.redalyc.org/html/2912/291221883005/

Submetido em 19 de agosto de 2019. Aprovado em 25 de setembro de 2019. 